\title{
Uso do plasma convalescente em pacientes graves pelo COVID-19: Revisão
}

\section{qualitativa de série de casos}

\author{
Use of convalescent plasma in serious patients by COVID-19: Qualitative case series review \\ Uso de plasma de convalecencia en pacientes graves por COVID-19: Revisión cualitativa de series \\ de casos
}

Recebido: 14/01/2021 | Revisado: 18/01/2021 | Aceito: 21/01/2021 | Publicado: 28/01/2021

\author{
Gabriel Couto Rocha \\ ORCID: https://orcid.org/0000-0001-5825-2540 \\ Universidade do Estado da Bahia, Brasil \\ E-mail: gabrielrocha.016@gmail.com \\ Aquiles Assunção Camelier \\ ORCID: https://orcid.org/0000-0001-5410-5180 \\ Universidade do Estado da Bahia, Brasil \\ E-mail: acamelier@uneb.br \\ Lohrany dos Santos Santana \\ ORCID: https://orcid.org/0000-0002-7400-5969 \\ Universidade do Estado da Bahia, Brasil \\ E-mail: lorhanny@live.com \\ Fernanda Warken Rosa Camelier \\ ORCID: https://orcid.org/0000-0003-2540-0142 \\ Universidade do Estados da Bahia, Brasil \\ E-mail: fcamelier@uneb.br \\ Roberto Rodrigues Bandeira Tosta Maciel \\ ORCID: https://orcid.org/0000-0002-4912-6005 \\ Universidade do Estado da Bahia, Brasil \\ E-mail: robertorbtm@hotmail.com
}

\begin{abstract}
Resumo
O objetivo desta pesquisa foi encontrar e avaliar a eficácia e a segurança do uso de plasma de pacientes recuperados para o tratamento de pacientes graves com COVID-19 (SARS-CoV-2), atraves de série de casos. A bsuca ocorreu nas bases de dados científicas como, Lilacs, MEDLINE, Google Academics, Scielo e Science Direct. Após a aplicação dos filtros para busca avançada, e do rastreamento por título, foi feito a leitura dos resumos pré-inclusos, depois avaliados na integra para extração de dados e síntese das informações e resultados. Ao final, três estudos observacionais do tipo série de casos foram incluídos. Os pacientes em estudo, estava usando madicamentos de forma paralela ao procedimento (ex.: antibioticios, antiviais e esteroides). Os pacientes, de forma geral, tiveram melhoras na maioria dos sintomas clinicos, como também, na diminuição dos títulos virais do SARS-CoV-2, nenhum evento adverso foi passivo de alerta. Conclue-se que as evidências encontradas neste trabalho são heterogênas e com bastante limitações, acerca de dose tranfundida, tempo de tratamento e critérios para elegir os pacientes, assim, devido as restrinções apresentadas, se faz necessiário realizações de ensaios clínicos com desenhos de estudo delineados, para certificar a segurança e efetividade do tratamento.
\end{abstract}

Palavras-chave: COVID-19; Plasma Convalescente; Terapia Imunológica; Segurança; Efetividade.

\begin{abstract}
The objective of this research was to find and evaluate the efficacy and safety of the use of plasma from recovered patients for the treatment of critically ill patients with COVID-19 (SARS-CoV-2), through a series of cases. The search took place in scientific databases such as Lilacs, MEDLINE, Google Academics, Scielo and Science Direct. After applying the filters for advanced search, and tracking by title, the pre-included abstracts were read, then evaluated in full for data extraction and synthesis of information and results. At the end, three case series observational studies were included. The patients in the study were using medications in a parallel way to the procedure (eg: antibiotics, antiviral drugs and steroids). Patients, in general, had improvements in most clinical symptoms, as well as in the decrease in SARS-CoV-2 viral titers, no adverse event was passive to alert. It is concluded that the evidences found in this work are heterogeneous and with a lot of limitations, about transfused dose, time of treatment and criteria to choose the patients, therefore, due to the presented restrictions, it is necessary to carry out clinical trials with outlined study designs, to certify the safety and effectiveness of the treatment.
\end{abstract}

Keywords: COVID-19; Convalescent plasma; Immunological therapy; Safety; Effectiveness. 


\section{Resumen}

El objetivo de esta investigación fue encontrar y evaluar la eficacia y seguridad del uso de plasma de pacientes recuperados para el tratamiento de pacientes críticos con COVID-19 (SARS-CoV-2), a través de una serie de casos. La búsqueda se realizó en bases de datos científicas como Lilacs, MEDLINE, Google Academics, Scielo y Science Direct. Después de aplicar los filtros para la búsqueda avanzada y el seguimiento por título, se leyeron los resúmenes pre-inclusos y luego se evaluaron en su totalidad para la extracción de datos y la síntesis de información y resultados. Al final, se incluyeron tres estudios observacionales de series de casos. Los pacientes del estudio estaban usando medicamentos de manera paralela al procedimiento (por ejemplo: antibióticos, medicamentos antivirales y esteroides). Los pacientes, en general, tuvieron mejorías en la mayoría de los síntomas clínicos, así como en la disminución de los títulos virales del SARS-CoV-2, ningún evento adverso fue pasivo a alerta. Se concluye que las evidencias encontradas en este trabajo son heterogéneas y con muchas limitaciones, en cuanto a dosis transfundida, tiempo de tratamiento y criterios para elegir a los pacientes, por lo que, debido a las restricciones presentadas, es necesario realizar ensayos clínicos con diseños de estudio perfilados, para certificar la seguridad y eficacia del tratamiento.

Palabras clave: COVID-19; Plasma de convalecencia; Terapia inmunológica; Seguridad; Eficacia.

\section{Introdução}

O COVID-19, também conhecido como coronavírus (vírus da família coronaviridae), é o principal causador de uma doença infecciosa respiratória aguda grave, tendo o SARS-CoV-2 (Severe Acute Respiratory Syndrome Coronavírus 2), como seu principal agente etiológico (Xu et al., 2020).

Em virtude da rapidez de instalação da pandemia associado a alta letalidade da infecção pela COVID -19, uma mudança no padrão na construção da evidência científica foi assumida pela comunidade científica, e uma série de estudos do tipo série de casos com diferentes intervenções plausíveis foram publicados nas bases de dados científicas, muitas vezes sem o tempo de seguimento ou o delineamento considerado clássico ( como por exemplo ensaios clínicos randomizados), em função da situação urgente instalada. Neste contexto, foi trazido à tona o uso do plasma convalescente como possibilidade terapêutica eficaz no tratamento da infecção pela COVID 19. O plasma convalescente possui mais de um século de uso, tendo sido utilizado quando ocorreram surtos de doenças infecciosas (a exemplo de H1N1, MERS, Gripe Espanhola - SUN, M. et al.,2020), sendo que foi aos poucos deixando de ser usado, com o surgimento de outros tratamentos e vacinas, que já poderiam ser produzidos em larga escala e possuíam eficiência semelhante, sem a "total" necessidade de pacientes recuperados. (Casadevall et al., 2020; Rubin et al., 2020). Na situação da pandemia da COVID a humanidade se defrontou com uma infecção de rápida e ampla instalação e mortalidade, com ausência de tratamento específico e ausência de prevenção como as vacinas, o que motivou a reutilização de um antigo recurso terapêutico como o plasma convalescente.

O plasma convalescente é a parte líquida do sangue, que possuem os anticorpos de combate a uma infecção, nesse caso o COVID-19, normalmente em casos como esse é retirado de pessoas já curada da infecção ou em processo de recuperação, sendo essa parte líquida do sangue coletada e posteriormente processado, para total reaproveitamento da parte necessária (anticorpos), sua administração passiva é a forma que pode fornecer imunidade imediata a pessoas susceptíveis. O plasma convalescente, na atual situação, é um produto de fácil acesso e de rápido processamento e aplicação, desde que haja um número suficiente de pacientes recuperados e habilitados a doação de sangue/plasma, que contenham títulos de anticorpos suficientes para reagir contra ao SARS-CoV-2. (Casadevall et al., 2020; Sullivan et al., 2020).

Devido a experiências no passado com surtos, como SARS-CoV-1, diversos estudos científicos têm surgido sobre o uso do plasma convalescente, sugerindo resultados promissores, mostrando que o soro convalescente pode neutralizar o vírus circulante antes de infectar a célula-alvo. O processo de neutralização ocorre, quando o anticorpo em especifico possui um antígeno-alvo do vírus, no caso SARS, tem a proteína S (Spike) que se localiza na superfície do vírus e é responsável pela ligação do SARS-CoV-2 às células epiteliais, incluindo pneumócitos, onde inicia o seu processo de infecção. Os anticorpos então, se ligam a essa proteína, impedindo que a mesma entre em contato com o alvo celular e o neutraliza impedindo sua ação. Para o vírus SARS-CoV-2, a forma de terapia passiva com anticorpos envolve o mesmo processo de neutralização da 
partícula viral, sendo atualmente testada, mesmo com o desenvolvimento de vacinas e pesquisada por algumas instituições de pesquisa em saúde que serão aqui abordadas.

Entretanto, existem problemas no que diz respeito aos resultados das atuais pesquisas com plasma convalescente, parte delas, em geral, originam de estudos não controlados, de braço único e com o tamanho limitado de pacientes, além de um desenho inconsistente dos estudos, isso acaba impedindo a comprovação real sobre a potencial efetividade desse tratamento, o que requer uma avaliação mais aprofundada na forma de ensaios clínicos sérios e preferencialmente randomizados. (Abdullah et al., 2020; Pessoa et al., 2020; Maxmen, 2020; Wu et al., 2020). Aqui neste trabalho, será apresentado prováveis benefícios da administração de plasma convalescente a pacientes com COVID-19 através de um resumo das evidências dessa estratégia na pandemia atual, relatado através de série de casos.

\section{Metodologia}

Trata-se de um estudo de revisão bibliográfica qualitativa (Pereira et al., 2018), de natureza descritiva, com a coleta realizada entre junho a outubro de 2020. Utilizando as palavras chaves; COVID-19; plasma convalescente; SARS-CoV-2; terapia imunológica. Foram incluídos no estudo artigos que redigissem de acordo com o objetivo da pesquisa, que é descrever a aplicação do plasma convalescente no tratamento do SARS-CoV-2, em pacientes internados graves, com desfecho de diminuição de tempo hospitalar e melhora dos sintomas clínicos, através de série de casos, caso controle ou relato de caso, apontando sua eficácia e segurança no uso em pacientes. Foram excluídos os artigos que não apresentaram informações relevantes sobre o tratamento com plasma convalescente, artigos de opinião, carta ao redator, dissertações de mestrado e monografias. O processo de seleção, extração de dados dos artigos e de identificação dos aspectos metodológicos foi realizado por dois revisores independentes. Quando havia discordância entre eles, os revisores liam novamente o artigo para reavaliação. As bases de dados, usadas na pesquisa foram: Lilacs (Literatura latina y del Caribe de Ciencias de la Salud), MEDLINE (através do PubMed), Google Academics, Scielo (Scientific Electronic Library Online) e Science Direct. Foram incluídos artigos em Português, Espanhol e Inglês.

Para inclusão no estudo, os pacientes doadores do plasma, foram anteriormente diagnosticados com COVID-19 (SARS-CoV-2) através do exame padrão ouro PCR-RT (reação em cadeia da polimerase em tempo real) e também testados para outros vírus e enfermidades (ex.: hepatite B e C, HIV e sífilis). E todos os pacientes, sem exceções, foram transfundidos com plasma convalescente e paralelamente estavam sendo administrados com antivirais, esteroides, antibióticos e antiinflamatórios.

A análise estatística do dos artigos foi feita por uma ferramenta para estudos observacionais do instituto Joanna Briggs (Aromataris et al., 2020). A pesquisa é feita através de um checklist, com avaliação crítica de série de casos, feita com análise de dez domínios metodológicos apresentados na tabela 1, (Aromataris et al., 2020).

\section{Resultados e Discussão}

Foram encontrados no total 1020 trabalhos nas bases de dados. Durante a triagem de títulos e resumos que não atenderam seleção, foram eliminados 1008 registros (que incluíam duplicatas). Após a leitura na integra dos pré-selecionados, 12 estudos foram incluídos na presente revisão, e somente 3 estudos atenderam os critérios de elegibilidade.

Os 3 estudos foram realizados em diferentes países, sendo, um nos Estados Unidos (Salazar et al., 2020) e dois na China (Duan et al., 2020; Shen et al.,2020). Todos tinham como objetivos descrever os efeitos do uso do plasma convalescente em pacientes internados pelo COVID-19 em estados grave, avaliando seus efeitos e o tempo de internação, além da diminuição dos sintomas como parte do desfecho. A média global dos estudos incluídos foram 7 de 10, como mostrado na Tabela 1, o que representa um valor aceitável para um estudo feito com pouco pacientes e algumas lacunas ao decorrer do seu processo (como 
exemplo, não houve o relato de se os casos foram feitos de forma consecutiva ou se houve tempo entre cada paciente).

Tabela 1. Ferramenta de avaliação da qualidade metodológica dos estudos inclusos no trabalho.

Critérios

\begin{abstract}
Definição explícita dos critérios de inclusão dos pacientes;
\end{abstract}
Mensuração da condição padronizada e confiável para todos os participantes;

\begin{tabular}{lccc}
$\begin{array}{l}\text { Utilização de métodos } \\
\text { válidos para identificação } \\
\text { da condição; }\end{array}$ & Sim & Sim & Sim \\
\hline $\begin{array}{l}\text { Inclusão consecutiva dos } \\
\text { participantes; }\end{array}$ & $\begin{array}{c}\text { Não relatado. Não há } \\
\text { informação sobre a } \\
\text { consecução dos casos }\end{array}$ & $\begin{array}{c}\text { Não relatado. Não há } \\
\text { informação sobre a } \\
\text { consecução dos casos }\end{array}$ & $\begin{array}{c}\text { Não relatado. Não há } \\
\text { informação sobre a } \\
\text { consecução dos casos }\end{array}$ \\
\hline $\begin{array}{l}\text { Inclusão completa de } \\
\text { todos os participantes no } \\
\text { período do estudo; }\end{array}$ & Não relatado & Não relatado & Não relatado \\
\hline $\begin{array}{l}\text { Características } \\
\text { dim }\end{array}$ & Sim & Sim & Sim
\end{tabular}

\section{demográficas dos}

participantes claramente

reportadas;

\begin{tabular}{lccc}
$\begin{array}{l}\text { Características clínicas } \\
\text { dos participantes } \\
\text { claramente reportadas; }\end{array}$ & Sim & Sim & Sim \\
\hline $\begin{array}{l}\text { Desfechos ou seguimento } \\
\text { dos resultados claramente } \\
\text { reportados; }\end{array}$ & $\operatorname{Sim}$ & $\operatorname{Sim}$ & $\mathrm{Sim}$ \\
\hline $\begin{array}{l}\text { Clareza nas informações } \\
\text { demográficas e clínicas; }\end{array}$ & $\operatorname{Sim}$ & Sim & Sim \\
\hline $\begin{array}{l}\text { Análise estatística } \\
\text { apropriada }\end{array}$ & Não aplicado & Não aplicado & Não aplicado \\
\hline Total & 7 de 10 & 7 de 10 & 7 de 10 \\
\hline
\end{tabular}

Fonte: JBI Manual adaptado traduzidas para o português.
Duan et al. (2020)

Salazar et al. (2020)

Shen et al. (2020) Sim Sim

Sim Sim $\quad$ Sim


condição não causada por transfusão de plasma. O tempo médio de internação pós-transfusão foi de 11 dias. 20 dos 25 pacientes haviam recebido alta ao final da pesquisa.

O segundo estudo foi realizado por Shen e colaboradores (2020), foi feito entre os meses de janeiro e março, analisando 5 pacientes no total ( 3 homens e 2 mulheres), sendo que um, apresentava duas comorbidades associadas (Insuficiência mitral e HAS) e 3 pacientes estavam acima dos 60, todos estava em estado grave, fazendo uso de ventilação mecânica e apresentavam a Síndrome do Desconforto Respiratório Agudo (SDRA), utilizando também terapia medicamentosa antiviral e esteroides. Além dos quadros já apresentados, para ser incluído como paciente grave e elegível para transfusão do plasma, eles precisavam apresentar: valores clínicos como $\mathrm{PaO} 2 / \mathrm{FIO} 2<300$, pneumonia grave com progressão rápida e carga viral em aumento exponencial. Durante os ensaios, foram transfundidos de 200 a $250 \mathrm{ml}$ de plasma convalescente, com o valor máximo de $400 \mathrm{ml}$. Como resultado clínico geral, todos os pacientes demonstraram melhora dos sintomas, no dia 3 póstransfusão, um paciente apresentou melhora da lesão pulmonar, o que também foi de resolução gradual nos outros pacientes, após o dia 3 pós-transfusão. No dia 12, pós transfusão, a febre havia diminuído para todos os pacientes, além da carga viral que também negativou em todos, em duas semanas 3 pacientes saíram da ventilação mecânica. $\mathrm{O}$ tempo de permanência hospitalar variou entre 51 e 55 dias, e no fim da pesquisa, 3 pacientes receberam alta neste mesmo período de tempo hospitalar.

O último estudo foi realizado entre janeiro e fevereiro, por Duan e colaboradores (2020), neste estudo eles incluíram 10 pacientes (seis homens e quatro mulheres), maiores que 18 anos (com idades entre 34 e 78 anos), e possuíam comorbidades associadas, tal qual eram o HAS e a doença cardiorrespiratória, que representavam, 3 e 1 paciente respectivamente. Para a inclusão precisavam seguir os critérios; falta de ar (dispneia), frequência respiratória em menos de 30 batimentos / min; nível de saturação de oxigênio no sangue ( $\mathrm{SatO} 2)$ em repouso, menos que $93 \%$ e pressão parcial de oxigênio ( $\mathrm{PaO} 2) / \mathrm{FIO} 2$ menor que $300 \mathrm{mmHg}$. Todos os pacientes estavam fazendo uso de terapia medicamentosa com antibióticos, antivirais e esteroides. $\mathrm{O}$ ensaio foi feito com apenas a transfusão única de $200 \mathrm{ml}$ de plasma convalescente e no momento da transfusão, todos apresentavam sintomas como febre, falta de ar, dor no peito e tosse, além de outros sintomas isolado. Os parâmetros para analise dos resultados da transfusão foram tidos através da verificação dos resultados clínicos, de imagem e laboratoriais. Sete dias após transfusão, todos os sintomas nos 10 pacientes, desapareceram ou melhoraram gradualmente. As primeiras melhoras foram, no após o dia 7 de transfusão, em que sete pacientes mostraram melhora nos exames radiológicos, com a absorção das lesões pulmonares e a não detecção da carga viral, houve também aumento da $\mathrm{SatO} 2$ em grande parte dos pacientes nos dias 1 e 3 após a transfusão. Nove em dez pacientes tiveram um aumento considerável da quantidade de anticorpos neutralizantes no soro, totalizando 1:640. No final da pesquisa, 7 pacientes continuaram internados até a finalização do estudo e apenas 3 pacientes receberam alta. Em geral todos os pacientes apresentaram melhora dos sintomas clínicos, nenhum óbito.

Assim como outros estudos (Cheng et al.,2020; Ye et al., 2020; Zhang et al.,2020) os resultados das pesquisas apresentadas apresentam uma linha de formação justificável acerca da aplicação do uso do plasma convalescente, embora possua significável resultados promissores é preciso cautela no seu andamento, já que ainda não há um estudo bem delineado acerca do uso do plasma convalescente (Anvisa-A, 2020; Anvisa-B, 2020). Mesmo bem tolerada, é necessário estar ciente dos diversos riscos e problemas transfusionais a serem ponderados durante os ensaios, a FDA (FDA, 2020) e a ANVISA (AnvisaA) relatam, que eventos adversos graves estão suscetíveis no processo. Precauções como a “... sobrecarga volêmica associada à transfusão (TACO) que possui alta mortalidade além dos riscos de transmissão de outras doenças infecciosas como HIV, HBC e HCV...”, por isso se faz necessário triagem sanguínea, não só de enfermidades, mas também de fator sanguíneo, sendo necessário que os doadores recuperados da COVID-19 sejam compatíveis e selecionados adequadamente, sendo submetidos a diversos mecanismos de avaliação (Ministério da Saúde, 2020).

Ainda também, não existe padronização quanto a quantidade utilizável de plasma convalescente, nem sobre o tempo pós-hospitalar para arrecadação do sangue dos doadores, uma nota técnica da ANVISA, Departamento de Atenção 
Especializada e Temática e da Coordenação-Geral de Sangue e Hemoderivados, indica uma recuperação clínica de 14 a 30 dias para ser elegível como doador, e 30 dias após recupação total apresentando diagnóstico clinico e laboratorial para aférese., em ambiente isolado das demais coletas para evitar contaminação cruzada com as demais coletas do hemocentro ou local especializado (Anvisa-C, 2020; Anvisa-D; 2020).

Em relação, ao uso do plasma e a todos os benefícios, precisam ser baseados em estudos de qualidade, mesmo em um contexto emergencial, é necessário a busca por pesquisas que testem a eficácia e segurança da imunoterapia, através de ensaios clínicos randomizados, cegos e aprovados por um conselho de ética (FDA, 2020; Ministério da Saúde, 2020), como prévia de possível resultados positivos, ao final das pesquisas, no total de 40 participantes dos estudos, 26 receberam alta no final da pesquisa.

As limitações identificadas nos estudos avaliados foram relacionadas ao delineamento dos estudos, o que dificultou concluir sobre a eficácia e segurança do uso do plasma convalescente em imunoterapias contra o COVID-19, além do número baixo de pacientes em cada caso, da melhora dos pacientes de forma questionável se era pela imunoterapia ou pela terapia medicamentosa e a falta de um grupo controle. Isso é discutido pelo fato de que a quantidade de material disponível acerca do COVID-19 seja bem limitada e da pouca existência até o momento de ensaios clínicos finalizados. Observou-se que existe uma heterogeneidade, dos trabalhos, no momento da transfusão do plasma, tanto sobre a quantidade e a frequência da transfusão, como da gravidade e da admissão hospitalar. Além de não houver a consistência dos estudos sobre o tempo de recupação dos pacientes doadores, nem do tempo entre a coleta e a transfusão, nem sobre a quantidade de anticorpos neutralizantes séricos disponíveis.

\section{Considerações Finais}

Esta revisão discorre que em todas as series de casos, após a administração do plasma convalescente houve uma melhora significativa na maior parte dos pacientes, mas devido as limitações apresentadas, se faz a necessidade de observações mais profundas através de ensaios clínicos. Uma das maiores limitações acerca disso é o baixo número de pacientes elegíveis para o estudo, embora o número de indivíduos infectados esteja diminuindo, muitos países estão passando por uma segunda onda e, portanto, é necessária uma consideração cuidadosa do desenho do estudo. Mesmo enfatizado que a prevalência do estudo ser de braço único, a maioria dos ensaios atestaram segurança na transfusão e baixo risco de efeitos colaterais, porém a testagem segura só pode ser feita através de ensaios clínicos randomizados, bem delineados, duplo-cego e que testifiquem a validade do método de forma eficaz. Considerando a situação atual, o uso do plasma convalescente se mostra potencialmente positivo no uso de ensaios clínicos de maneira que fortaleça os dados na esperança de proteger a vida dos pacientes receptores. É possível compreender que o estudo de caso, é um passo inicial para criação de um estudo randomizado com resultados confiáveis, lembrando que quaisquer conclusões tiradas com base em nesses dados são de valor limitado e essas conclusões estão sujeitas a mudança à medida que resultados mais confiáveis se tornam disponíveis.

Assim como sugestão, criar como desfecho secundário de ensaios, a qualidade de vida dessas pessoas, devido a exposição de qualquer risco que elas venham a passar, não só físico como psicológico, juntamente com trabalhos que avaliem efetividade comparando com medicamentos em tratamento paliativo que já atestaram eficácia em tratar sintomas, mostrando resultados através da diminuição das taxas de internações hospitalares por COVID-19 ou a não progressão da doença a nível de UTI.

\section{Referências}

Abdullah, S. F., Anees, R. \& Alaa, M. G. (2020). Adaptive Immune Responses to SARS-CoV-2 and Prophylactic Vaccines for COVID-19. GSJ: Volume 8, Issue 5 . 
Anvisa-A. Agência Nacional de Vigilância Sanitária. (2020). N N 19/2020/SEI/GSTCO/DIRE1/ANVISA. Nota Técnica, Aspectos regulatórios do uso de plasma de doador convalescente para tratamento da Covid-19. https://docs.bvsalud.org/biblioref/2020/05/1096174/plasmaconvalescente-covid19.pdf.

Anvisa-B. Agência Nacional de Vigilância Sanitária. (2020). No 21/2020-CGSH/DAET/SAES/MS. Nota Técnica, Coleta e transfusão de plasma de convalescentes para uso experimental no tratamento de pacientes com COVID-19. http://www.mpgo.mp.br/portal/arquivos/2020/08/28/17 _14_32_355_Nota_T\%C3\%A9cnica_n_21_2020_CGSH_DAET_SAES_MS_Orientacoes_Plasma_convalescente_COVID_19.pdf.

Anvisa-C. Agência Nacional de Vigilância Sanitária. (2000). Resolução de Diretoria Colegiada - RDC No 73, DE 3 DE AGOSTO DE 2000. Dispõe sobre o Programa Nacional de Sangue e Hemoderivados, regula o uso e a disponibilidade do Plasma Fresco Congelado Excedente do Uso Terapêutico no Brasil e dá outras providências. http://bvsms.saude.gov.br/bvs/saudelegis/anvisa/2000/res0073_03_08_2000.html.

Anvisa-D. Agência Nacional de Vigilância Sanitária. (2014). Resolução da Diretoria Colegiada - RDC nº 34 , de 11 de junho de 2014 . Dispõe sobre as Boas Práticas no Ciclo do Sangue. https://saude.rs.gov.br/upload/arquivos/carga20170553/04145350-rdc-anvisa-34-2014.pdf.

Aromataris, E., Munn, Z., Barker, T., Moola, S., Tufanaru, C., Stern, C., McArthur, A. \& Stephenson, M. (2020). Methodological quality of case series studies, JBI Evidence Synthesis.

Casadevall, A. \& Pirofski, L. (2020). The convalescent sera option for containing COVID-19. J Clin Invest, 130(4):1545-1548.

Cheng, Y., Wong, R., Soo, Y. O. Y., Wong, W. S., Lee, C. K., Ng, M. H. L., Chan, P., Wong, K. C., Leung, C. B. \& Cheng, G. (2020). Use of convalescent plasma therapy in SARS patients in Hong Kong. Eur J Clin Microbiol Infect Dis. 24: 44-46.

Duan, K., Liu, B., Li, C., Zhang, H., Yu, T., Qu, J., Zhou, M., Chen, L., Meng, S., Hu, Y., Peng, C., Yuan, M., Huang, J., Wang, Z., Yu, J., Gao, X., Wang, D., Yu, X., Li, L., Zhang, J., Wu, X., Li, B., Xu, P., Chen, W., Peng, Yan., Hu, Y., Lin, L., Liu, X., Huang, S., Zhou, Z., Zhang, Wang, Y., Zhang, Z., Deng, Z., Xia, Z., Gong, Q., Zhang, W., Zheng, X., Liu, Y., Yang, H., Zhou, D., Yu, D., Hou, J., Shi, Z., Chen, S., Chen, Z., Zhang, X. \& Yang, X. (2020). Effectiveness of convalescent plasma therapy in severe COVID-19 patients. Proceedings of the National Academy of Sciences of USA (PNAS). https://www.pnas.org/content/117/17/9490.

Food and Drug Administration (FDA). (2020). Recommendations for Investigational COVID-19 Convalescent Plasma. Development \& Approval Process (CBER). https://www.fda.gov/vaccines-blood-biologics/investigational-new-drug-ind-or-device-exemptionide-process cber/recommendations-investigationalcovid-19-convalescent-plasma.

Maxmen, A. (2020). How blood from coronavirus survivors might save lives. A Nature Research Journal (Nature). https://www.nature.com/articles/d41586020-00895-8.

Ministério da Saúde do Brasil. (2020). Coordenação De Gestão De Protocolos Clínicos e Diretrizes Terapêuticas. Diretrizes Para Diagnóstico e Tratamento Da Covid-19, Versão 4. Secretaria de Ciência, Tecnologia, Inovação e Insumos Estratégicos em Saúde - SCTIE. https://saude.rs.gov.br/upload/a rquivos/202004/14140600-2-ms-diretrizes-covid-v2-9-4.pdf.

Pereira, A. S., Shitsuka D. M., Parreira F. J. \& Shitsuka, R. (2018). Metodologia da pesquisa científica. [e-book]. Santa Maria. Ed. UAB/NTE/UFSM. https://repositorio.ufsm.br/bitstream/handle/1/15824/Lic_Computacao_Metodologia-Pesquisa-Cientifica.pdf?sequence=1.

Pessoa, B. M. S., Barroso, C. T., Vila, D. J. C., Barbosa, E. M. L., Seffair, I. A., Melo, J. V. O., Becil, J. N., Rodrigues, N. M. V., Freitas, P. H. A. G., Rocha, R. B., Bentes, T. M., Rodrigues, T. F. \& Santos, M.C. (2020). Imunoterapias No Tratamento Da Covid-19. Revista Desafios. 7. COVID-19.

Rubin, R. (2020). Testing an Old Therapy Against a New Disease: Convalescent Plasma for COVID-19. JAMA. 323(21).

Salazar, E., Perez, K., Ashraf, M., Chen, J., Castillo, B., Christensen, P. A., Eubank, T., Bernard, D. W., Eagar, T. N., Long, S. W., Subedi, S., Olsen, R. J., Leveque, C., Schwartz, M. R., Dey, M., Chavez-East, C., Rogers, J., Shehabeldin, A., Joseph, D., Williams, G., Thomas, K., Masud, F., Talley, C., Dlouhy, K. G., Lopez, B. V., Hampton, K., Lavinder, J., Gollihar, J. D., Maranhão, A. C., Ippolito, G. C., Saavedra, M. O., Cantu, C. C., Yerramilli, P., Pruitt, L., \& Musser, J. M. (2020). Treatment of Coronavirus disease 2019 patients with convalescent plasma reveals a signal of significantly decreased mortality. The American Journal of Pathology, 190, 2290-2303.

Shen, C., Wang, Z., Zhao, F., Yang, Y., Li, J., Yuan, J., Wang, F., Li, D., Yang, M., Xing, Li., Wei, J., Xiao, H., Yang, Y., Qu, J., Qing, L., Chen, L., Xu, Z., Peng, L., Li, Y., Zheng, H., Chen, F., Huang, K., Jiang, Y., Liu, D., Zhang, Z., Liu, Y. \& Liu, L. (2020). Treatment of 5 Critically Ill Patients With COVID-19 With Convalescent Plasma. JAMA.

Sullivan, H. C. \& Roback, J. D. (2020). Convalescent Plasma: Therapeutic Hope or Hopeless Strategy in the SARS-CoV-2 Pandemic. Transfusion Medicine Reviews. 34. 145-150.

Sun, M., A potentially effective treatment for COVID-19: A systematic review and meta-analysis of convalescent plasma therapy in treating severe infectious disease. International Journal of Infectious Diseases, 98, 334-346.2020.

Wu, F., Zhao, S., Yu, B., Chen, Y. M., Wang, W., Song, Z. G., Hu, Y., Tao, Z. W., Tian, J. H., Pei, Y. Y., Yuan, M. L., Zhang, Y. L., Dai, Y. L., Wang, Q. M., Zheng, J. J., Xu, L., Holmes, E. C., \& Zhang, Y. Z. (2020). A new coronavirus associated with human respiratory disease in China. Nature, 579, $265-269$.

Ye, M., Fu, D., Ren, Y., Wang, F., Wang, D., Zhang, F., Xia, X. \& Lv, T. (2020). Treatment with convalescent plasma for COVID-19 patients in Wuhan, China. J Med Virol.1-12.

Xu, X., Chen, P., Wang, J., Feng, J., Zhou, H., Li, X., Zhong, W. \& Hao, P. (2020). Evolution of the novel coronavirus from the ongoing Wuhan outbreak and modeling of its spike protein for risk of human transmission. Sci China Life Sci, 63, 457-460.

Zhang, B., Liu, S., Tan, T., Huang, W., Dong, Y., Chen, L., Chen, O., Zhang, L., Zhong, Q., Zhang, X., Zou, Y. \& Zhang, S. (2020). Treatment with Convalescent Plasma for Critically Ill Patients with Severe Acute Respiratory Syndrome Coronavirus 2 Infection. CHEST, 158(1): 9-13. 